\title{
Sequential trans-dimensional Monte Carlo for range-dependent geoacoustic inversion
}

\author{
Jan Dettmer ${ }^{\text {a) }}$ and Stan E. Dosso \\ School of Earth and Ocean Sciences, University of Victoria, Victoria, British Columbia, Canada V8W 3P6 \\ Charles W. Holland \\ Applied Research Laboratory, The Pennsylvania State University, State College, Pennsylvania 16804-0030
}

(Received 9 November 2010; revised 25 January 2011; accepted 28 January 2011)

\begin{abstract}
This paper develops a sequential trans-dimensional Monte Carlo algorithm for geoacoustic inversion in a strongly range-dependent environment. The algorithm applies advanced Markov chain Monte Carlo methods in combination with sequential techniques (particle filters) to carry out geoacoustic inversions for consecutive data sets acquired along a track. Changes in model parametrization along the track (e.g., number of sediment layers) are accounted for with trans-dimensional partition modeling, which intrinsically determines the amount of structure supported by the data information content. Challenging issues of rapid environmental change between consecutive data sets and high information content (peaked likelihood) are addressed by bridging distributions implemented using annealed importance sampling. This provides an efficient method to locate high-likelihood regions for new data which are distant and/or disjoint from previous high-likelihood regions. The algorithm is applied to simulated reflection-coefficient data along a track, such as can be collected using a towed array close to the seabed. The simulated environment varies rapidly along the track, with changes in the number of layers, layer thicknesses, and geoacoustic parameters within layers. In addition, the seabed contains a geologic fault, where all layers are offset abruptly, and an erosional channel. Changes in noise level are also considered. (c) 2011 Acoustical Society of America. [DOI: 10.1121/1.3557052]
\end{abstract}

PACS number(s): 43.30.Pc, 43.30.Ma, 43.60.Pt, 43.60.Wy [AIT] $\quad$ Pages: 1794-1806

\section{INTRODUCTION}

Prediction of shallow-water transmission loss uncertainty intrinsically relies on knowledge of the spatial variability and uncertainty of the sediment geoacoustic parameters along the track of interest. Knowledge of sediment spatial variability and uncertainty also plays a crucial role in understanding and predicting reverberation and clutter. Most geoacoustic-inversion work to date has focused on one-dimensional environmental models that average the environment laterally over the seabed-footprint area of the experiment which can range from $\sim 100 \mathrm{~m}$ (Refs. 1 and 2) to many kilometers. ${ }^{3-7}$ Some work has examined the spatial variability of sediment parameters by inverting multiple data sets collected at sparse sampling locations along a track..$^{8-10}$ A central issue here that is sometimes overlooked is that variability can only be examined meaningfully once parameter uncertainties are properly and quantitatively understood at each sampling location. ${ }^{10}$

Model parametrization (e.g., the number of seabed layers) plays a fundamental role in obtaining meaningful environmental uncertainty estimates. ${ }^{4,10-12}$ The Bayesian information criterion has been applied to address model parametrization selection ${ }^{11}$ but represents a point estimate [computed at the maximum a posteriori (MAP) estimate] which may not adequately represent the model space. In a

\footnotetext{
a) Author to whom correspondence should be addressed. Electronic mail: jand@uvic.ca.
}

more rigorous and quantitative approach, Bayesian evidence (the likelihood of the model gives the measured data) can be computed $^{4,12}$ using methods such as annealed importance sampling ${ }^{13}$ (AIS). Bayesian evidence does not rely on point estimates but takes into account the full posterior probability distribution (PPD) of the problem. However, computations of evidence and point estimates thereof require carrying out inversions for each individual model in a group of models of interest.

To further generalize the model selection approach, Dettmer et al. ${ }^{14}$ applied a trans-dimensional partition model parametrization to geoacoustic inversion, where the number of unknowns in the problem is itself an unknown. Transdimensional inversion is an efficient and effective approach to model selection, since the fixed-dimensional sub-space of each allowed model parametrization is sampled proportional to its marginal likelihood. Hence, the uncertainty about the choice of environmental parametrization is accounted for in the inversion in a hierarchical Bayesian sense. ${ }^{15}$ In such an approach, the environmental model is regularized by the data, which limit the overall amount of the structure and determine where the structure is supported over the depth interval of interest. ${ }^{14,16}$ The amount of structure allowed can vary with the depth. For example, gradients in sound velocity may be approximated by the ensemble structure of several interfaces spread over some parts of the partition model while other areas can have homogeneous structure and sharp discontinuities characterized by ensembles of interfaces confined tightly in depth. 
Sequential Bayesian Monte Carlo (SMC) methods ${ }^{17,18}$ are commonly applied to follow PPDs of interest (target distributions) as they evolve over time such as in source tracking $^{19-21}$ and a wide range of other applications where data are continuously accumulated over time. ${ }^{18}$ In this context, "time" is used as a general term to index the evolution of a target in some way and is not limited to actual time but could also refer to, for example, space. Since the target distribution evolves in time, such problems are commonly termed dynamic Bayesian systems, and following the target distribution, is generally referred to as tracking. In such problems, pure Markov chain Monte Carlo $3,22-24$ (MCMC) methods can be prohibitively expensive computationally since chains need to be restarted (including burn-in) whenever the existing data set is augmented by a new data. In contrast, SMC updates an existing probability distribution to follow its evolution over time while new data are introduced, with the parameter space allowed to expand or contract, depending on the problem. Sequential Monte Carlo algorithms typically involve a set of points in the state space (referred to as a cloud of particles) that is propagated from one data set to the next and updated according to the additional data information that arrives over time. As an application of this general methodology, Yardim et al. ${ }^{25}$ applied a particle filter to a simulated matched-field inversion example where data were accumulated over time and showed that geoacoustic target distributions can be successfully tracked while significantly reducing computational effort compared to separate geoacoustic inversions carried out for individual data sets. Yardim et $a l .{ }^{21}$ also applied a sequential importance resampling filter (the bootstrap filter ${ }^{17}$ ) to a matched-field tracking problem where a low-dimensional geoacoustic model and a source model were tracked over time.

SMC methods are computationally more challenging when the parameter values change substantially between steps and/or when the data are very informative (i.e., tightly constrain parameters). ${ }^{18}$ When the change in parameter values between steps is large (e.g., when parameters of interest are highly variable), the measured data will typically also change rapidly between steps. This results in a target distribution that is difficult to follow since particle filters rely on parameter estimates for past data sets to provide starting parameter estimates for current data. This problem is greatly exacerbated when the data are also highly informative: If the data information content is high, the likelihood is very peaked; that is, the posterior mass of interest is concentrated in a small volume of the state space and is difficult to find. ${ }^{26}$ In such cases, particle filters such as the bootstrap filter can fail as the particle cloud rapidly degenerates and is not able to follow the target. To address these issues, a wide range of MCMC methods have been applied in SMC algorithms to add sample diversity to the particle cloud as well as to assist in tracking peaked likelihoods for highly informative dynamic Bayesian systems. ${ }^{18,20}$

This paper develops a particle filter that utilizes several advanced MCMC methods to track a trans-dimensional target distribution for highly informative acoustic data with peaked likelihoods in a rapidly changing ocean environment. The particle filter accounts for changes in the sediment layering structure, including changes in the number of layers, with a reversible jump Markov chain Monte Carlo (rjMCMC) partition model. ${ }^{14}$ In addition, problems of peaked likelihood and sample impoverishment ${ }^{20}$ are addressed by introducing bridging distributions to connect target distributions at the previous and current steps. The bridging is accomplished using AIS $^{12,13}$ to define a series of interpolating distributions and computing importance weights along each annealing trajectory which are used in the resampling step of the particle filter. ${ }^{26}$ Resampling with replacement is then carried out according to the weights of the importance sampling distribution given by the series of interpolating distributions.

The algorithm is applied here to simulated seabed reflection-coefficient data as a function of frequency and angle. The specific experiment geometry corresponds to an acoustic source and horizontal line array (HLA) towed at a fixed altitude above the water-sediment interface. The simulation represents a large number of 170 consecutive data sets that here represent a track length of several kilometers. A different realization of normally distributed, random noise is added for each ping. The seabed environment for this simulation varies rapidly along the track, with changes to the number of layers, layer thicknesses, and geoacoustic parameters within layers. In addition, the seabed contains a geologic fault, where all layers are offset abruptly, and an erosional channel. Changes in the noise level on the data, including pings with poor data quality, are also considered.

\section{SEQUENTIAL MONTE CARLO AND BAYESIAN INFERENCE}

Bayesian inference on a dynamic system, where the PPD evolves as additional data are acquired over time, is a challenging problem. Classic MCMC methods are often inappropriate as computational demand can be prohibitively high. SMC methods have been developed to address Bayesian inference on dynamical systems in a computationally feasible manner by estimating the posterior distribution recursively. This section formulates the basic Bayesian framework for the SMC algorithm described in Sec. III based on Doucet et al., ${ }^{18}$ where a more detailed treatment can be found. The observed data in a dynamical system are acquired over time $t$ which can be considered a natural number $t \in \mathbb{N}$. The accumulated data up to time $t$ are $\mathbf{d}_{1: t}=\left(\mathbf{d}_{1}, \ldots, \mathbf{d}_{t}\right)$. The unknown parameters of interest (commonly referred to as hidden parameters or hidden states) $\mathbf{x}_{t}$ can be treated as a Markov process with an initial probability distribution $P\left(\mathbf{x}_{0}\right)$ and transition density $P\left(\mathbf{x}_{t} \mid \mathbf{x}_{t-1}\right)$ from state $t-1$ to state $t$. The parameters also accumulate over time such that $\mathbf{x}_{0: t}=\left(\mathbf{x}_{0}, \ldots, \mathbf{x}_{t}\right)$. The PPD at time $t$ can then be expressed with Bayes' theorem as

$$
P\left(\mathbf{x}_{0: t} \mid \mathbf{d}_{1: t}\right)=\frac{P\left(\mathbf{x}_{0: t}\right) P\left(\mathbf{d}_{1: t} \mid \mathbf{x}_{0: t}\right)}{\int P\left(\mathbf{x}_{0: t}\right) P\left(\mathbf{d}_{1: t} \mid \mathbf{x}_{0: t}\right) d \mathbf{x}_{0: t}} .
$$

The parameter vector $\mathbf{x}_{0: t}$ containing all unknowns of the dynamic system up to time $t$ increases in dimension rapidly with time. Hence, the target distribution Eq. (1) (also 
referred to as the smoothing density) also expands rapidly and becomes intractable for many problems. Sequential methods address this problem by recursively estimating the target, in particular, by estimating the marginal filtering distribution $P\left(\mathbf{x}_{t} \mid \mathrm{d}_{1: t}\right)$. Equation (1) can be expressed recursively as

$$
P\left(\mathbf{x}_{0: t} \mid \mathbf{d}_{1: t}\right)=P\left(\mathbf{x}_{0: t-1} \mid \mathbf{d}_{1: t-1}\right) \frac{P\left(\mathbf{d}_{t} \mid \mathbf{x}_{t}\right) P\left(\mathbf{x}_{t} \mid \mathbf{x}_{t-1}\right)}{P\left(\mathbf{d}_{t} \mid \mathbf{d}_{1: t-1}\right)},
$$

where new data are introduced through the likelihood $P\left(\mathbf{d}_{t} \mid \mathbf{x}_{t}\right)$ and the denominator is the normalizing constant. The filtering distribution can be recursively expressed as

$$
\begin{aligned}
& P\left(\mathbf{x}_{t} \mid \mathbf{d}_{1: t-1}\right)=\int P\left(\mathbf{x}_{t} \mid \mathbf{x}_{t-1}\right) P\left(\mathbf{x}_{t-1} \mid \mathbf{d}_{1: t-1}\right) \mathbf{d} \mathbf{x}_{t-1}, \\
& P\left(\mathbf{x}_{t} \mid \mathbf{d}_{1: t}\right)=\frac{P\left(\mathbf{x}_{t} \mid \mathbf{d}_{1: t-1}\right) P\left(\mathbf{d}_{t} \mid \mathbf{x}_{t}\right)}{\int P\left(\mathbf{x}_{t} \mid \mathbf{d}_{1: t-1}\right) P\left(\mathbf{d}_{t} \mid \mathbf{x}_{t}\right) \mathbf{d} \mathbf{x}_{t}}
\end{aligned}
$$

These recursions are the basis of sequential Bayesian methods and must be estimated numerically (e.g., SMC) for nonlinear non-Gaussian systems. Equation (3) can be considered the predictive step and Eq. (4) the update step that takes newly arrived data into account through the likelihood $P\left(\mathbf{d}_{t} \mid \mathbf{x}_{t}\right)$.

Inference on a dynamic Bayesian system can be carried out with the predictive and update equations using particle filters. Let $\left\{\mathbf{x}_{0: t}^{(i)} ; i=1, \ldots, N_{p}\right\}$ denote a group of points or particles drawn independently from the smoothing distribution $P\left(\mathbf{x}_{0: t} \mid \mathbf{d}_{1: t}\right)$. In many cases, independent draws from $P\left(\mathbf{x}_{0: t} \mid \mathbf{d}_{1: t}\right)$ are not possible and an importance sampling function $\pi\left(\mathbf{x}_{0: t} \mid \mathbf{d}_{1: t}\right)$ is introduced [which must include support for $P\left(\mathbf{x}_{0: t} \mid \mathbf{d}_{1: t}\right)$ but can otherwise be arbitrary] from which independent samples are drawn. Importance weights for particle $i$ are computed as

$$
\omega\left(\mathbf{x}_{0: t}^{(i)}\right)=\frac{P\left(\mathbf{x}_{0: t} \mid \mathbf{d}_{1: t}\right)}{\pi\left(\mathbf{x}_{0: t} \mid \mathbf{d}_{1: t}\right)}
$$

and normalized as $\tilde{\omega}_{t}^{(i)}=\omega\left(\mathbf{x}_{0: t}^{(i)}\right) 0 / \sum_{j=1}^{N_{p}} \omega\left(\mathbf{x}_{0: t}^{(j)}\right)$. The particle approximation to the target is then given by

$$
P\left(\mathbf{x}_{0: t} \mid \mathbf{d}_{1: t}\right) \approx \sum_{i=1}^{N_{p}} \tilde{\omega}_{t}^{(i)} \delta\left(\mathbf{x}_{1: t}-\mathbf{x}_{1: t}^{(i)}\right)
$$

where $\delta$ is the Dirac delta function. However, all data $\mathbf{d}_{1: t}$ are needed to carry out the importance sampling and the importance sampling function must be rewritten to carry out recursive estimation:

$$
\pi\left(\mathbf{x}_{0: t} \mid \mathbf{d}_{1: t}\right)=\pi\left(\mathbf{x}_{0: t-1} \mid \mathbf{d}_{1: t-1}\right) \pi\left(\mathbf{x}_{t} \mid \mathbf{x}_{0: t-1}, \mathbf{d}_{1: t}\right) .
$$

Hence, the importance weights can be written recursively as

$$
\tilde{\omega}_{t}^{(i)}=\tilde{\omega}_{t-1}^{(i)} \frac{P\left(\mathbf{d}_{t} \mid \mathbf{x}_{t}^{(i)}\right) P\left(\mathbf{x}_{t}^{(i)} \mid \mathbf{x}_{t-1}^{(i)}\right)}{\pi\left(\mathbf{x}_{t}^{(i)} \mid \mathbf{x}_{0: t-1}^{(i)}, \mathbf{d}_{1: t}\right)}
$$

Using the prior distribution as the importance distribution yields the particular case of

$$
\pi\left(\mathbf{x}_{0: t} \mid \mathbf{d}_{1: t}\right)=P\left(\mathbf{x}_{0: t}\right)=P\left(\mathbf{x}_{0}\right) \prod_{k=1}^{t} P\left(\mathbf{x}_{k} \mid \mathbf{x}_{k-1}\right),
$$

resulting in weights

$$
\tilde{\omega}_{t}^{(i)}=\tilde{\omega}_{t-1}^{(i)} P\left(\mathbf{d}_{t} \mid \mathbf{x}_{t}^{(i)}\right)
$$

which is widely used as the simplest form of sequential importance sampling. However, pure sequential importance sampling such as this can cause a degenerate particle cloud even for small $t$, since new data information will cause only very few particles to carry significant weight, while most other particles carry near-zero weight. This problem is particularly prominent when new data introduce parameter information that changes estimates substantially from previous values. Hence, sequential importance sampling is generally extended by resampling (weighted resampling with replacement $^{27,28}$ ), which was later introduced to SMC methods in the bootstrap filter. ${ }^{17} \mathrm{~A}$ resampling operation multiplies particles with high weights and deletes particles with low weights, resulting in an equally weighted particle cloud that concentrates in the high-likelihood region of the state space. This method is particularly attractive when only the filtering distribution $P\left(\mathbf{x}_{t} \mid \mathbf{d}_{1: t}\right)$ is of interest, since the path of each particle through time need not be stored. The bootstrap filter can be computationally costly for many problems, and many extensions to this basic filter have been published. ${ }^{18,20,29}$

\section{SEQUENTIAL TRANS-DIMENSIONAL BAYESIAN INVERSION}

This section gives the theoretical background for the different Monte Carlo strategies used here to formulate an efficient trans-dimensional particle filter for tracking rapidly changing sediment environments.

\section{A. Bridging distributions for resampling}

SMC methods are most efficient when the dynamic system changes slowly enough between data sets that there is a significant overlap between the prediction and target distributions. However, typical sediment layering structures can exhibit sudden and large changes due to geological events such as faults or erosional channels filled in with material that differs significantly from the surrounding sediments. Sensitivity to environmental variations is greatest for experimental methods that have a small footprint on the seafloor and hence do not average over large seabed areas. Further, multi-frequency reflection-coefficient data collected on a HLA close to the seabed can have very high information content and low data errors since environmental effects such as oceanographic variability do not play an important role. Hence, this type of data is challenging to analyze using SMC methods due to the high data information content and the possibility that new data may introduce significantly different environmental information such that the particles from a previous step may not be representative of new data. These 
difficulties are exacerbated when trans-dimensional inverse problems are considered or multi-modal target distributions are encountered.

The issues described above are also encountered in geoacoustic inversion for highly nonlinear stationary systems that do not evolve over time but have strongly multi-modal posterior distributions where modes are separated by extensive low likelihood regions. ${ }^{12}$ For such problems the simulated annealing heuristic ${ }^{30}$ was developed to address optimization problems with many posterior modes and avoid getting trapped in local minima. In simulated annealing, a sequence of probabilities $P_{j}(x) \propto P_{0}(x)^{\beta_{j}}$ is defined, where $\beta_{j} \in[0,1]$ is often referred to as the inverse temperature. The algorithm "anneals" by moving through the sequence of interpolating distributions, increasing $\beta$ from 0 to 1 . Acceptance of moving from one state to the next is decided according to Eq. (15), with low $\beta$ values causing the algorithm to move freely between isolated modes but becoming increasingly confined with increasing $\beta$. However, simulated annealing does not provide unbiased sampling of the PPD.

AIS (Refs. 12 and 13) combines ideas of simulated annealing, MCMC, and importance sampling to assign importance weights to many simulated annealing runs. As the number of runs increases, the end points of the annealing trajectories weighted by the importance weights provide an unbiased sample of the PPD. In a sequential framework, AIS can be used to provide highly effective bridging distributions from the prediction distribution to the filtering distribution (the target at time $t$ ), and this paper applies AIS in this way as proposed in Godsill and Clapp. ${ }^{26}$ The bridging is defined through a sequence of interpolating distributions, such as

$$
f_{l}\left(\mathrm{x}_{t}\right)=\pi\left(\mathrm{x}_{t}\right)^{1-\beta_{l}} P\left(\mathrm{x}_{t} \mid \mathbf{d}_{1: t}\right)^{\beta_{l}},
$$

where the algorithm proceeds from $\beta_{L}$ to $\beta_{0}$ with $0=\beta_{L}<\beta_{L-1}<\cdots<\beta_{0}=1$ and $\pi\left(\mathbf{x}_{t}\right)$ is the importance sampling distribution which can be set to $\pi\left(\mathbf{x}_{t}\right)=P\left(\mathbf{x}_{t} \mid \mathbf{x}_{t-1}\right)$ $P\left(\mathbf{x}_{t-1} \mid \mathbf{d}_{1: t-1}\right)$, giving a sequence of interpolating distributions that gradually introduce the information of new data through the likelihood $P\left(\mathbf{d}_{1: t} \mid \mathbf{x}_{t}\right)$

$$
f_{l}\left(\mathbf{x}_{t}\right)=P\left(\mathbf{x}_{t-1} \mid \mathbf{d}_{1: t-1}\right) P\left(\mathbf{x}_{t} \mid \mathbf{x}_{t-1}\right) P\left(\mathbf{d}_{1: t} \mid \mathbf{x}_{t}\right)^{\beta_{l}} .
$$

In addition, MCMC operators are used that leave each distribution in the sequence invariant. ${ }^{13}$ The associated weight $\omega^{(i)}$ for particle $\mathbf{x}^{(i)}$ is computed along the trajectory as

$$
\omega^{(i)}=\prod_{l=0}^{L-1} \frac{f_{l}\left(\mathbf{x}_{t}^{(i)(l)}\right)}{f_{l+1}\left(\mathbf{x}_{t}^{(i)(l)}\right)} .
$$

This scheme uses the sequence of bridging distributions $f_{l}$ to move the particle cloud from one step to the next and improves the particles convergence to the new high-likelihood region which may be distant and disjoint from the past high-likelihood region. The resulting particle cloud approximates the filtering distribution $P\left(\mathbf{x}_{t} \mid \mathbf{d}_{1: t}\right)$ by a weighted sample, and the weights can be used for resampling operations. In addition, resampling can be applied at any point along the trajectory, but this is not done here since it is expected to degrade performance on a parallel computer.

\section{B. Trans-dimensional partition model}

Sequential inference for spatially varying sediment properties must account for changes in the layering structure, including events where new layers appear or disappear over the depth of interest. Dettmer et al. ${ }^{14}$ showed that such model selection problems can be addressed efficiently in a trans-dimensional formulation, ${ }^{31}$ meaning that the number of unknown parameters is itself an unknown hyper-parameter that is integrated over in a hierarchical Bayesian sense, resulting in a posterior that spans multiple parameter spaces of different dimensions. A key advantage of the trans-dimensional approach is that the posterior includes the uncertainty due to the limited knowledge of the model parametrization.

Let $d \in \mathbb{R}^{N}$ be a random variable consisting of $N$ observed data containing information about a physical system. Further, let $\mathcal{I}_{k}$ denote a group of models specifying particular choices of physical theory, model parametrizations, and error statistics to explain that system, where $k \in \mathcal{K}$ and $\mathcal{K}$ is a countable set. Let $\mathbf{m}_{k} \in \mathbb{R}^{M_{k}}$ be a random variable of the $M_{k}$ free parameters representing a realization of model $\mathcal{I}_{k}$. Green ${ }^{15}$ shows that Bayes' rule can be written for a Bayesian hierarchical model to include parameter $k$

$$
P\left(k, \mathbf{m}_{k} \mid \mathbf{d}\right)=\frac{P(k) P\left(\mathbf{d} \mid k, \mathbf{m}_{k}\right) P\left(\mathbf{m}_{k} \mid k\right)}{\sum_{k^{\prime} \in \mathcal{K}} \int_{M} P\left(k^{\prime}\right) P\left(\mathbf{d} \mid k^{\prime}, \mathbf{m}_{k^{\prime}}^{\prime}\right) P\left(\mathbf{m}_{k^{\prime}}^{\prime} \mid k^{\prime}\right) \mathbf{d m}_{k^{\prime}}^{\prime}},
$$

where $k$ can be interpreted as indexing possible choices of models (as commonly used in model selection via evidence or point estimates thereof) or as variable dimension parameter of a single model that spans several multi-dimensional spaces. In Eq. (14) $P(k)$ is the prior over the models in $\mathcal{K}$ to be considered. The state variables of the inversion for the trans-dimensional case are then given by $\mathbf{x}=\left(k, \mathbf{m}_{k}\right)$ and are of dimension $M_{k}$. The state space is trans-dimensional and given by the union of all fixed-dimensional spaces in $\mathcal{K}$, i.e., $\bigcup_{k \in \mathcal{K}}\left(\{k\} \times \mathbb{R}^{M_{k}}\right)$. A Markov chain that samples this state space can be defined which converges to the trans-dimensional joint posterior $P\left(k, \mathbf{m}_{k} \mid \mathbf{d}\right)$ (see Sec. III B 1).

A partition modeling approach is applied here to parametrize the positions of sediment interfaces in the sediment model extending from the seafloor to a maximum depth of interest. Perturbing one interface in a partition model only changes the neighboring two layers and leaves the rest of the model invariant; this is consistent with the idea that relatively small perturbations to the model result in a higher probability of accepting the perturbation as a new state in a MCMC algorithm. The perturbations are generally small compared to the distance to the nearest interface neighbor, and the crossing of interfaces is not allowed. Inference on the partition model PPD can be carried out with ensemble averages over the sediment structure. The results are similar to regularized inversions ${ }^{32}$ but without the requirement of specifying subjective regularization terms (such as requiring 
model smoothness). ${ }^{14,33}$ The number of sediment interfaces is given by the model index $k$ in Eq. (14).

\section{Reversible jump MCMC}

A Markov chain that samples the trans-dimensional posterior in Eq. (14) must be able to undergo dimension changes (jumps) in the state space while not violating the requirement of detailed balance (the probability of a step from a current state to a new state must be equal to the probability of reversing the step) to ensure unbiased sampling. ${ }^{34}$ In the fixed-dimension case $\left(k=k^{\prime}\right)$, the Metropolis-Hastings (MH) algorithm can be used to sample from the posterior by implementing the $\mathrm{MH}$ acceptance criterion for a step from the current model $\mathbf{m}_{k}$ to a proposed model $\mathbf{m}_{k^{\prime}}^{\prime}$

$$
\alpha=\min \left[1, \frac{P\left(\mathbf{m}_{k^{\prime}}^{\prime}\right)}{P\left(\mathbf{m}_{k}\right)}\left(\frac{P\left(\mathbf{d} \mid \mathbf{m}_{k^{\prime}}^{\prime}\right)}{P\left(\mathbf{d} \mid \mathbf{m}_{k}\right)}\right)^{\beta} \frac{Q\left(\mathbf{m}_{k} \mid \mathbf{m}_{k^{\prime}}^{\prime}\right)}{Q\left(\mathbf{m}_{k^{\prime}}^{\prime} \mid \mathbf{m}_{k}\right)}\right],
$$

where $\beta$ is an annealing parameter (see Sec. III A) that can be considered unity here. The proposal distribution $Q$ is centered on the current model $\left(\mathbf{m}_{k}\right)$ and is used to propose a model $\mathbf{m}_{k^{\prime}}^{\prime}$ as a new state of the Markov chain. In this paper, the proposal distribution is chosen to be Gaussian, however, other choices are possible. Green ${ }^{15,31}$ shows that a rjMCMC algorithm can be implemented similar to the $\mathrm{MH}$ algorithm by generalizing the $\mathrm{MH}$ acceptance to apply to a step from the current model $\left(k, \mathbf{m}_{k}\right)$ to a proposed model $\left(k^{\prime}, \mathbf{m}_{k^{\prime}}^{\prime}\right)$, where $k^{\prime}$ can be different from $k$, as

$$
\alpha=\min \left[1, \frac{P\left(\mathbf{m}_{k^{\prime}}^{\prime}\right)}{P\left(k, \mathbf{m}_{k}\right)}\left(\frac{P\left(\mathrm{~d} \mid k^{\prime}, \mathbf{m}_{k^{\prime}}^{\prime}\right)}{P\left(\mathrm{~d} \mid k, \mathbf{m}_{k}\right)}\right)^{\beta} \frac{Q\left(k, \mathbf{m}_{k} \mid k^{\prime}, \mathbf{m}_{k^{\prime}}^{\prime}\right)}{Q\left(k^{\prime}, \mathbf{m}_{k^{\prime}}^{\prime} \mid k, \mathbf{m}_{k}\right)}|\mathbf{J}|\right],
$$

where $|\mathbf{J}|$ is the determinant of the Jacobian for a diffeomorphism from $\left(k, \mathbf{m}_{k}\right)$ to $\left(k^{\prime}, \mathbf{m}_{k^{\prime}}^{\prime}\right)$ (see Ref. 14 for details). The most commonly applied form of rjMCMC is the birth-death scheme, ${ }^{14,34}$ in which case the determinant of the Jacobian is unity. The scheme must be implemented in a manner that ensures unbiased sampling and equal probability of birth and death moves to maintain detailed balance. In this scheme, a birth move is defined to be the introduction of new parameters. For example, in the partition model a new layer can be created by introducing a new interface at a random position in the seabed model, increasing the model indexing parameter $k$ by one. Alternatively, a death move randomly picks and deletes an interface, reducing $k$ by one. This paper implements the birth-death approach such that the geoacoustic model is represented as $\left(k, \mathbf{m}_{k}\right)=(k, \mathbf{z}, \boldsymbol{\gamma}$, $\boldsymbol{\sigma})$, where $\mathbf{z}$ denotes the sediment-depth partitioning, $\gamma$ contains the geoacoustic properties of all layers (i.e., $\gamma=(\mathbf{c}, \boldsymbol{\rho}$, $\boldsymbol{\alpha})$, where $\mathbf{c}, \boldsymbol{\rho}$, and $\boldsymbol{\alpha}$ are vectors of sound velocities, densities, and attenuations, respectively). Expressing the sediment model in terms of interface depths $(\mathbf{z})$ and geoacoustic layer parameters $\gamma$ is useful to implement the rjMCMC algorithm. Note that this is done without loosing any generality with regard to the geoacoustic model. The data-error model is denoted $\boldsymbol{\sigma}$.

\section{MCMC prior information}

The prior information used in this paper consists of bounded, uniform probability distributions with the range of parameter values chosen to represent physically reasonable values and set wide enough that the data predominantly determine the posterior. Since $\left(k, \mathbf{m}_{k}\right)=(k, \mathbf{z}, \boldsymbol{\gamma}, \boldsymbol{\sigma})$, the prior probability is $P(k) P\left(\mathbf{m}_{k} \mid k\right)=P(k) P(\mathbf{z} \mid k) P(\boldsymbol{\gamma} \mid k) P(\boldsymbol{\sigma} \mid k)$. In particular, the prior distribution for the model index parameter is given by (assuming equal preference for all models)

$P(k)= \begin{cases}\left(k^{\max }-k^{\min }\right)^{-1} & \text { if } k^{\min } \leq k \leq k^{\max } \\ 0 & \text { else. }\end{cases}$

The prior distribution for $\gamma$ is given by

$P(\gamma \mid k)= \begin{cases}\prod_{i=1}^{3(k+1)}\left(\gamma_{i}^{\max }-\gamma_{i}^{\min }\right)^{-1} & \text { if } \gamma_{i}^{\min } \leq \gamma_{i} \leq \gamma_{i}^{\max } \\ 0 & \text { else, }\end{cases}$

and the prior distribution for the data-error model is given by

$P(\boldsymbol{\sigma} \mid k)= \begin{cases}\prod_{i=1}^{N_{\sigma}}\left(\sigma_{i}^{\max }-\sigma_{i}^{\min }\right)^{-1} & \text { if } \quad \sigma_{i}^{\min } \leq \sigma_{i} \leq \sigma_{i}^{\max } \\ 0 & \text { else, }\end{cases}$

where superscripts min and max indicate the lower and upper bounds applied. The $\sigma_{i}$ refers to the $N_{\sigma}$ parameters of the data-error model which may include data-error standard deviations and parametric expressions for serial data-error correlations (such as autoregressive parameters). The prior distribution for $\mathbf{z}$ is ${ }^{34}$

$$
P(\mathbf{z} \mid k)=\left[\frac{K !}{k !(K-k) !}\right]^{-1}
$$

which can be shown to simplify when formulating the birthand death-acceptance ratios such that the temporarily assumed grid dimension $K$ cancels. ${ }^{14}$

\section{TRANS-DIMENSIONAL PARTICLE FILTER WITH BRIDGING}

In this section, the components outlined in Secs. III A and III B are implemented in a particle filter to estimate the filtering distribution along a track where the dimension of the environmental model can change and large, abrupt changes in parameters can be addressed. Bridging distributions based on AIS are applied between the predictive and filtering distributions to address the abrupt changes and peaked likelihoods that are typical for sediment environments and reflection-coefficient data. The algorithm is illustrated schematically in Fig. 1 and implemented as follows. The filter is initialized with an equally weighted particle cloud $\left\{\mathbf{x}_{0}{ }^{(i)(k)}, \omega_{0}{ }^{(i)}, i=1, \ldots, N_{p}\right\}$, where $k$ indicates the 


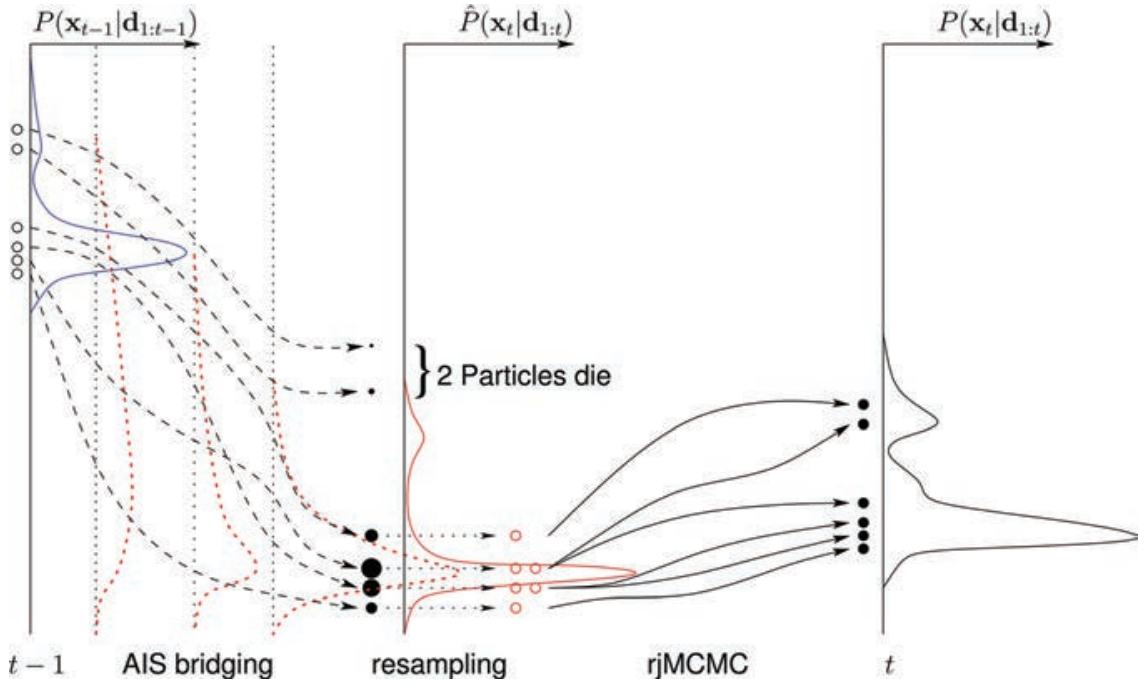

FIG. 1. (Color online) Concept of the reversible jump particle filter with bridging distributions for six particles. Bridging distributions are indicated as dotted lines, AIS trajectories and rjMCMC paths are indicated by arrows. The size of points indicates the weight associated with a particle. model index parameter. The initial particle cloud is taken from a large sample of $P\left(\mathbf{x}_{0} \mid \mathbf{d}_{0}\right)$, obtained from a rjMCMC inversion that applies strong chain thinning ${ }^{23}$ (intermittently saving samples from the chain, to reduce serial correlation between saved samples). The particle cloud at step $t-1$ is then propagated through the algorithm by gradually introducing the new data information $P\left(\mathbf{d}_{t} \mid \mathbf{x}_{t}\right)$ through a sequence of bridging distributions $\left\{f_{l}, l=0, \ldots, L\right\}$ as discussed in Sec. III A. The resulting particle cloud $\left\{\mathbf{x}_{t}^{(i)(k)}, \omega_{t}{ }^{(i)}\right\}$ is an approximate, weighted sample $\hat{P}\left(\mathbf{x}_{t} \mid \mathbf{d}_{1: t}\right)$ of the target $P\left(\mathbf{x}_{t} \mid \mathbf{d}_{1: t}\right)$. Resampling can then be carried out for this weighted sample.

The number of interpolating distributions and the scaling determined by the $\beta_{l}$ sequence is chosen dynamically to ensure that a wide range of difficult transitions between steps can be accommodated while maintaining high efficiency when the dynamic system does not change significantly between steps. An iterative approach is used that starts with a small number of interpolating distributions and increases the number of distributions, as needed, until the resulting particle cloud reaches a sufficiently high-likelihood region. This target likelihood region is chosen to $\mathrm{be}^{35}$

$$
\tilde{P}\left(\mathbf{d}_{t} \mid \mathbf{x}_{t}\right)=\left(\frac{1}{(2 \pi)^{N / 2} \sigma_{t-1}^{N}} \exp (N / 2)\right) \pm \sqrt{2 N}
$$

where $\sigma_{t-1}$ is the mean data standard deviation obtained at step $t-1$ and $N$ is the total number of data in $\mathbf{d}_{t-1}$. When a sufficiently large number of particles (arbitrarily set to 5\%) is in that target region, the bridging iteration is considered successful and resampling is carried out. Resampling is carried out for each dimension $k$ to preserve the distribution over $k$.

After resampling, a rjMCMC chain is carried out for each particle to further balance the particle cloud to the new filtering distribution $P\left(\mathbf{x}_{t} \mid \mathbf{d}_{1: t}\right)$, which concludes one step for the particle filter. A challenging aspect of the problem is trans-dimensional mixing of the chains, and a sufficiently long rjMCMC chain must be run to allow acceptance of several birth and death moves. If the chain is too short, the algorithm may not balance properly to the new target when the number of layers changes between steps.

\section{INVERSION EXAMPLES}

This section applies the trans-dimensional sequential inversion algorithm developed in Secs. III and IV to simulated reflection-coefficient data along a strongly range-dependent track. The geometry of the simulation is chosen such that each data set collected along the track consists of 32 reflection-coefficient measurements ${ }^{36}$ over a grazingangle range of $28^{\circ}-66^{\circ}$ at 4 frequency bands with center frequencies of $1000,1200,2000$, and $2400 \mathrm{~Hz}$ with a $25-\mathrm{Hz}$ bandwidth. Such data could, for example, be collected with a source and HLA towed by a ship, see Fig. 2. The angular range is chosen here to be relatively small to represent practicable experimental setups with towed arrays.

\section{A. Range-dependent environment}

The simulated seabed environment is modeled after typical sediments that are found in many shallow-water settings and are of interest for transmission loss prediction. Shallowwater seabeds have been shown to exhibit ${ }^{10}$ range

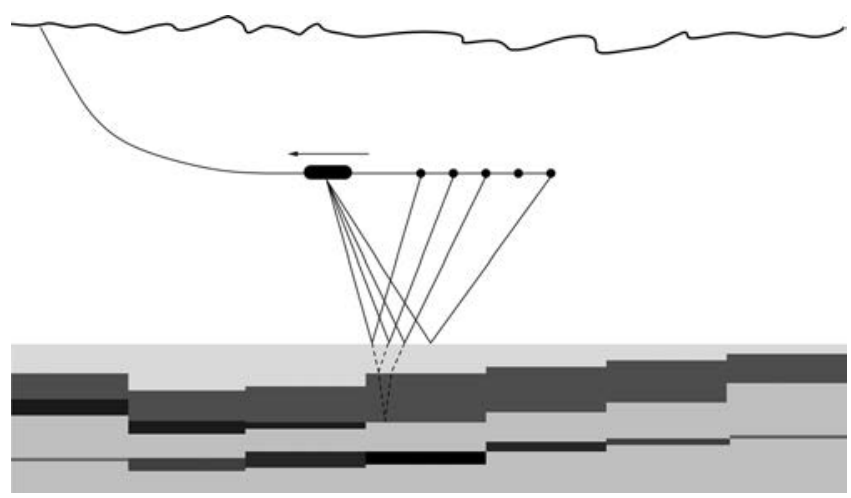

FIG. 2. Experimental setup used to acquire spatial geoacoustic data using a towed source and array configuration. 
dependence in terms of the number of layers, layer thicknesses as well as changes of geoacoustic parameters within layers. The track considered here is sampled by 170 consecutive data sets which are identified in the following by their ping number, since a typical application with a towed array would include a towed sound source that generates a signal ("ping") at fixed time intervals. Each data set represents a spatially confined part of the seabed that is considered range independent. In an experiment with a towed array, pings could occur at intervals as small as a few meters. The spatial structure in the simulation was designed with a ping separation of order $10 \mathrm{~m}$. The true environment used to generate the data involves a complicated range-dependent seabed, where all parameters change along the track. The true environment is shown in Fig. 3 and includes both smooth variations in geoacoustic properties along the track as well as several large and abrupt changes. The uppermost layer of soft sediment thins slowly along the track while at the same time an underlying high-velocity layer also changes in thickness and increases in velocity by $100 \mathrm{~m} / \mathrm{s}$. Between pings 28 and 70 , a thin high-velocity $(\sim 1610 \mathrm{~m} / \mathrm{s})$ sediment lens is introduced below three thicker layers. From ping 45 to 46, a geological fault is simulated, where all layers drop $60 \mathrm{~cm}$ and the topmost $60 \mathrm{~cm}$ are filled in by a low-velocity sediment not discernible from the layer immediately below. The velocity in the upper layer then increases gradually along the track until the layer pinches out at ping 124. Most layer interface depths also have random variability of a few centimeters from ping to ping. The overall number of layers (not counting the basement) starts with $k=3$ layers at ping 1 , then increases to $k=6$ at the fault, decreases to $k=2$ at ping 124 , and again increases to $k=3$. A second prominent feature is an erosional channel at the water-sediment interface from ping 148 to 154 . Here, the relatively high-velocity sediment is eroded and the channel is filled in with low-velocity sediment. The change is abrupt, increasing the number of layers by one and causing a dramatic change in layer thickness for the higher velocity sediment. Finally, a rapid change in layer thickness occurs after ping 127 in layer 3, where the layer thickness decreases by $20 \mathrm{~cm}$ and then abruptly increases again by the same amount over a series of 6 pings. Throughout the model, the density changes in a manner consistent with the sound velocity ${ }^{37}$ but attenuation variation is chosen to be simpler since the sensitivity of acoustic reflectivity data to attenuation is generally much lower than to velocity and density.

Data were computed for the environmental model in Fig. 3 using a recursive plane-wave reflection-coefficient model $^{38}$ and random Gaussian errors with standard deviation of 0.033 were added to each data set. The standard deviation was chosen to be representative of errors typically observed for measured data by the authors. Data for four selected pings are shown in Fig. 4, highlighting the significant changes the fault and erosional channel cause in the data. The overall fit of the particle cloud as represented by 200 randomly drawn particles and $95 \%$ highest probability density (HPD) credibility intervals for the replica data for all particles are also shown. The fit to the data is good relative to the noise level, and the algorithm properly fits the data even after large, abrupt environmental changes. Such changes require the particle cloud to access distant parts of the data space as well as properly balance the particles in high-likelihood regions of the state space that are separated from previous locations of high target likelihood by extensive low-likelihood regions.

\section{B. Posterior inference}

The sequential Monte Carlo algorithm is applied to the data described in Sec. V A using a fairly large set of 10000 particles, since the primary interest here is to estimate posterior probability (filtering) distributions for each ping. If only mean parameter estimates and standard deviations are of interest, the number of particles could be much smaller. ${ }^{39}$ The final result of the geoacoustic inversion along the track is a sequence of 170 trans-dimensional posterior distributions, which capture the data information content for the sediment

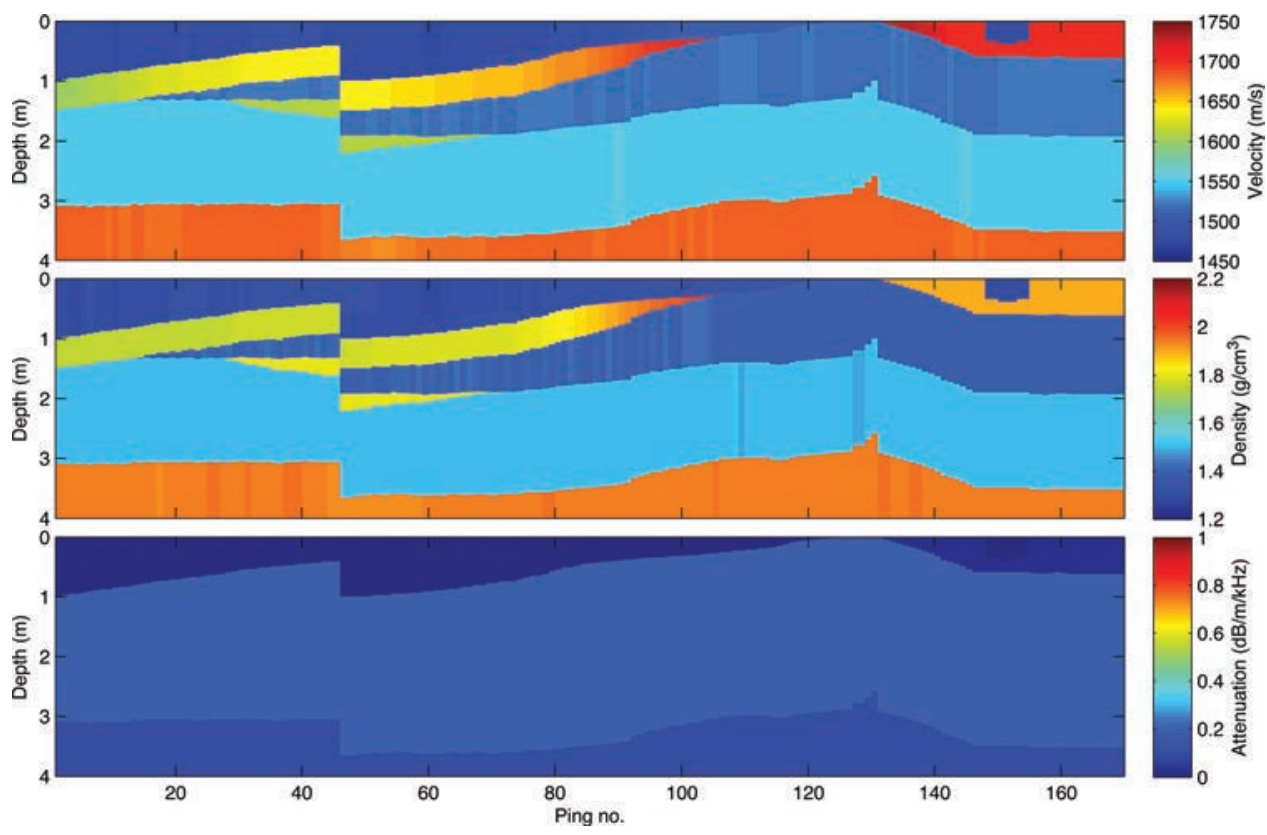

FIG. 3. (Color online) The true 170ping environment for the simulation. 


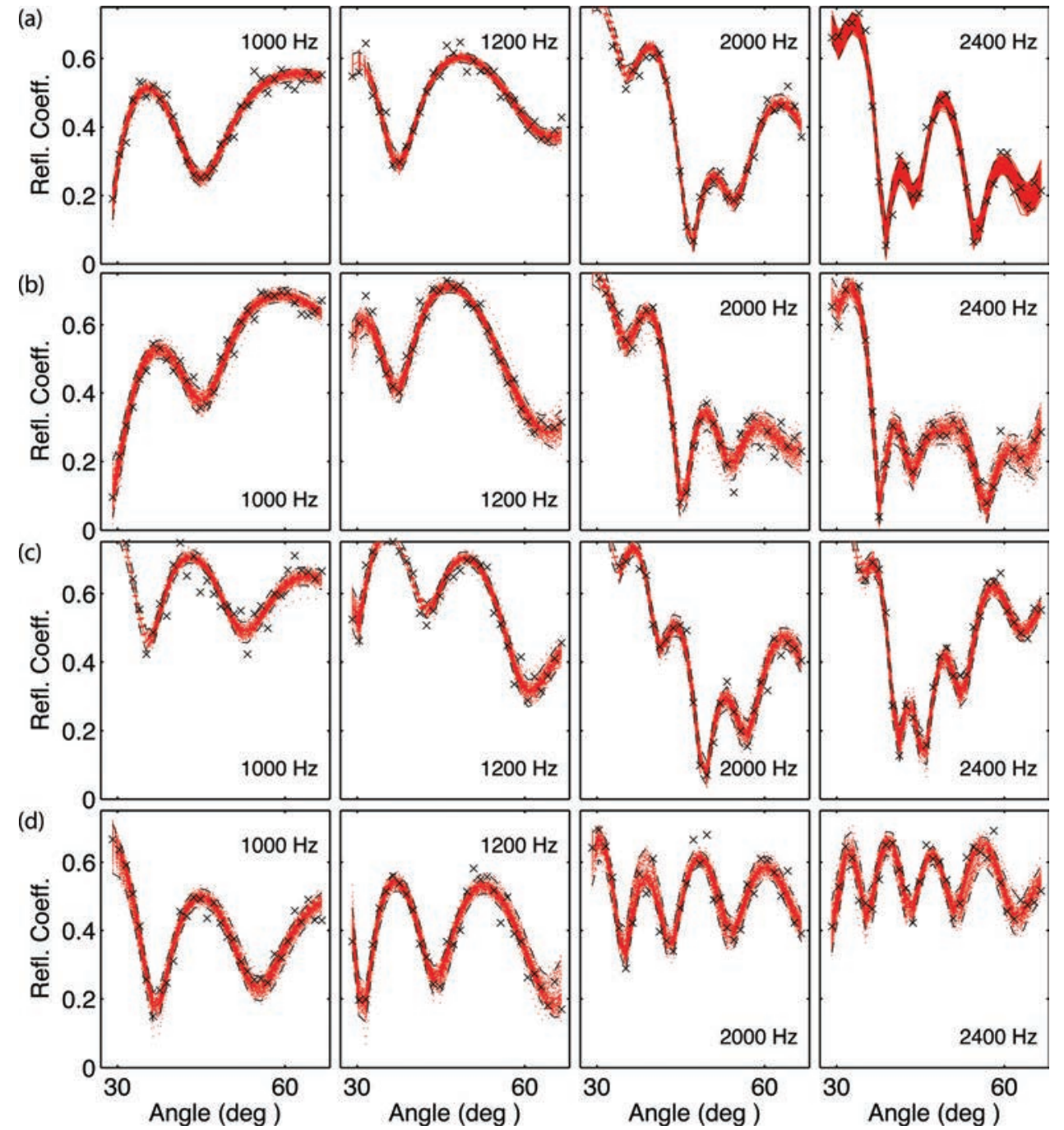

FIG. 4. (Color online) Simulated reflection-coefficient data (crosses) and ensemble fit (dotted lines) for pings before and after the fault event [pings (a) 45 and (b) 46], and before and within the erosional channel [pings (c) 146 and (d) 152]. Also shown are the 95\% HPD credibility intervals for the predicted data for the entire particle cloud (dashed).

structure as sampled by the reflection-coefficient data. This section explores some of the possibilities to carry out inference on these distributions. In particular, inference is carried out for trans-dimensional partition model ensembles that quantify geoacoustic parameter uncertainty as a function of depth and on interface probabilities as a function of depth and ping resulting in interface probability maps. Figure 5(a) shows the marginal probability for the number of sediment interfaces or layers (the model indexing parameter $k$ ) along the track. The inversion results closely track the true number of interfaces; however, in some instances, where small impedance contrasts between layers exist, the maximum of the k-probability underestimates the true number of interfaces (as it should due to the natural parsimony of the method). An

(a)
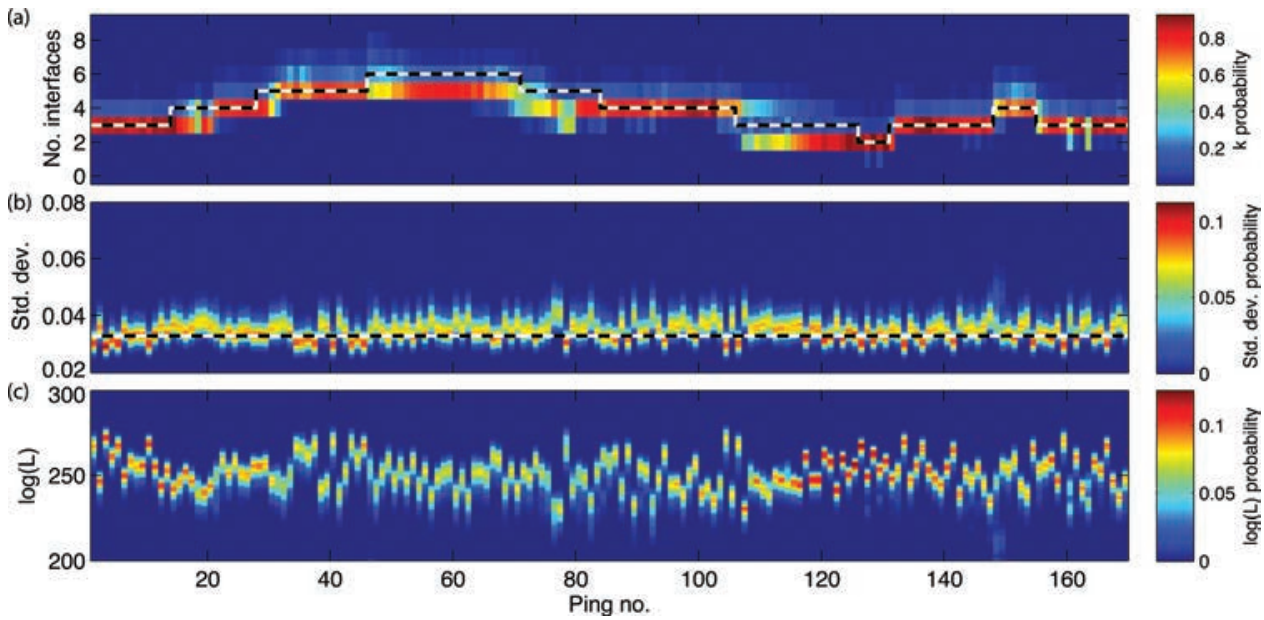

FIG. 5. (Color online) Marginal probability distributions along the track for (a) number of interfaces and (b) data standard deviation (true number of layers and standard deviations are given as dashed lines). (c) The log-likelihood along the track. 


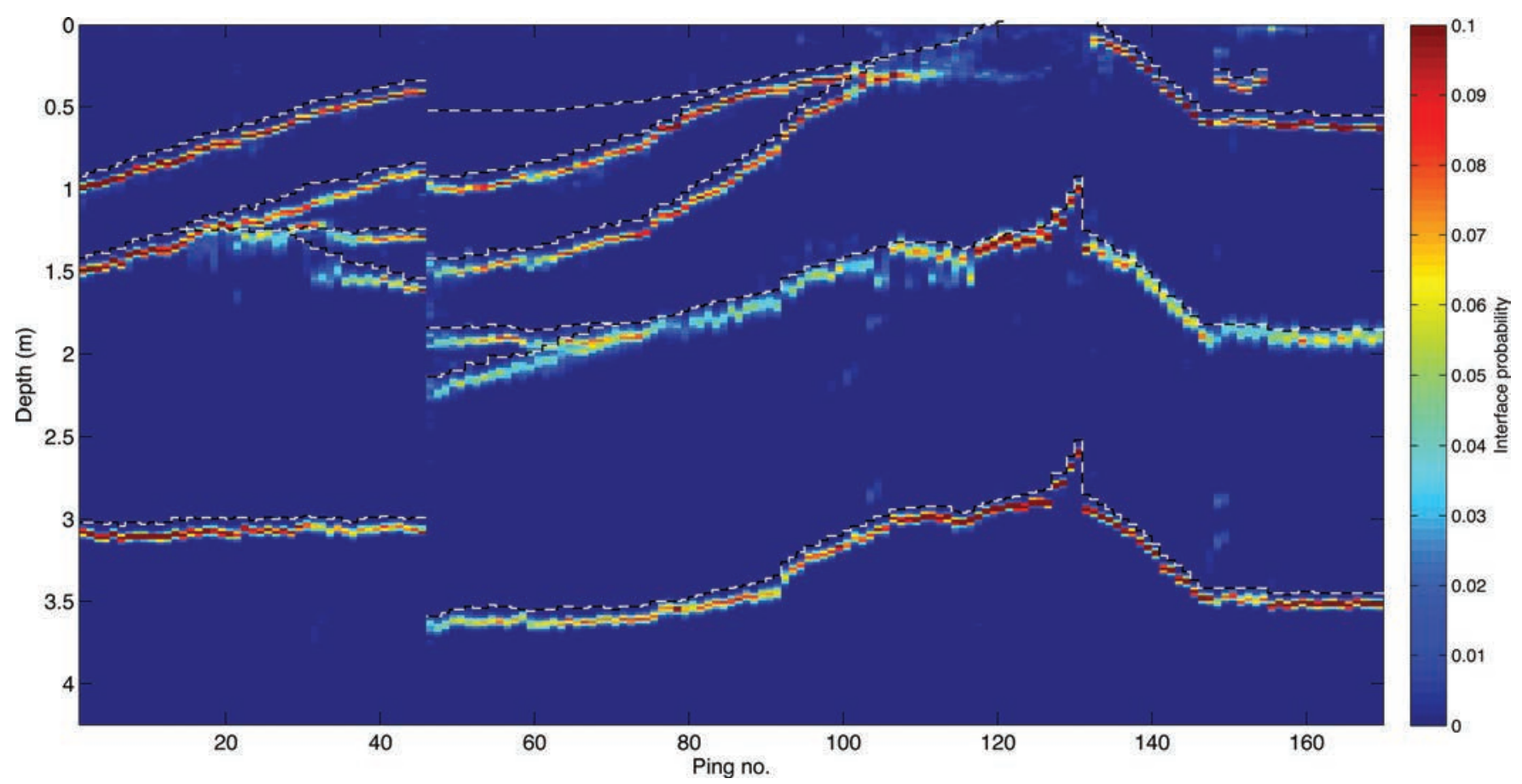

FIG. 6. (Color online) Interface-depth probability distributions along the track. True interface locations are shown as dashed lines which are offset slightly for display purposes.

example of this occurs just after the fault event (ping 46), where the top $60 \mathrm{~cm}$ of sediment are filled in with a material of very similar sound velocity and density to that below it. Marginals for the data standard deviation, which is treated as an unknown in the problem, are shown in Fig. 5 (b) and indicate that the inversion results along the track are close to the true values used in the simulation. Log-likelihood marginals are shown in Fig. 5(c). The results in Figs. 5(b) and (5c) indicate that the inversion is stable along the track and that a reasonable fit to the data is achieved for all pings.

Figure 6 shows the probability of interfaces as a function of depth along the track. This form of marginalization recovers layering structure in a manner similar to sub-bottom profilers. However, unlike standard sub-bottom profilers, Fig. 6 represents a quantitative map of true interface-depth probabilities that does not require an arbitrary scaling velocity to convert two-way time to sub-bottom depth. In addition, the interface-depth probability also indicates the sharpness of the discontinuity (i.e., the size of the impedance contrast and the depth interval over which it occurs). The results show that interfaces are successfully identified by the partition model and are tracked from ping to ping where supported by the data.

Figure 7 compares the posterior mean model results for velocity and density [Figs. 7(a) and 7(c), respectively] to the true model for the corresponding parameters [Figs. 7(b) and 7(d)].
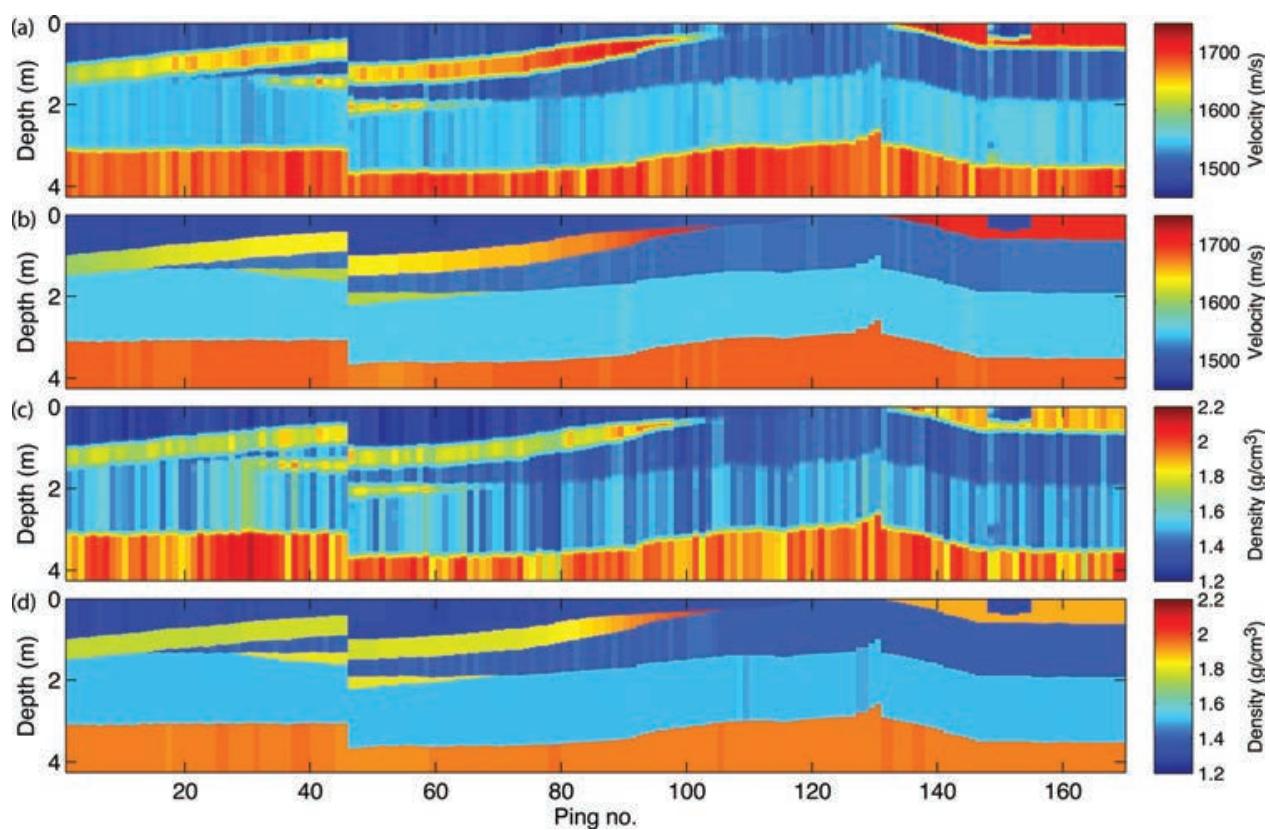

FIG. 7. (Color online) Inversion results in terms of posterior mean models for (a) velocity and (c) density along the track compared to true (b) velocity and (d) density structures. 
The mean models provided by the inversion algorithm are in excellent agreement with the true model and recover the compete structure of the true model, including the fault and erosional channel events. In addition, some more subtle environmental variations are successfully tracked, including the slow increase in velocity and density in the thin highvelocity layer until it pinches out at ping 105 . The thin highvelocity lens between pings 28 and 70 is also recovered with good velocity and density estimates. When the new layer appears along the track (a large change in information content), the algorithm needs a few pings to fully incorporate the new information in the posterior. Once the layer is established, the algorithm tracks it well until it pinches out.

The inversions carried out along the range-dependent track result in posterior distributions that allow for detailed inference of various parameter properties, including parameter

(a)
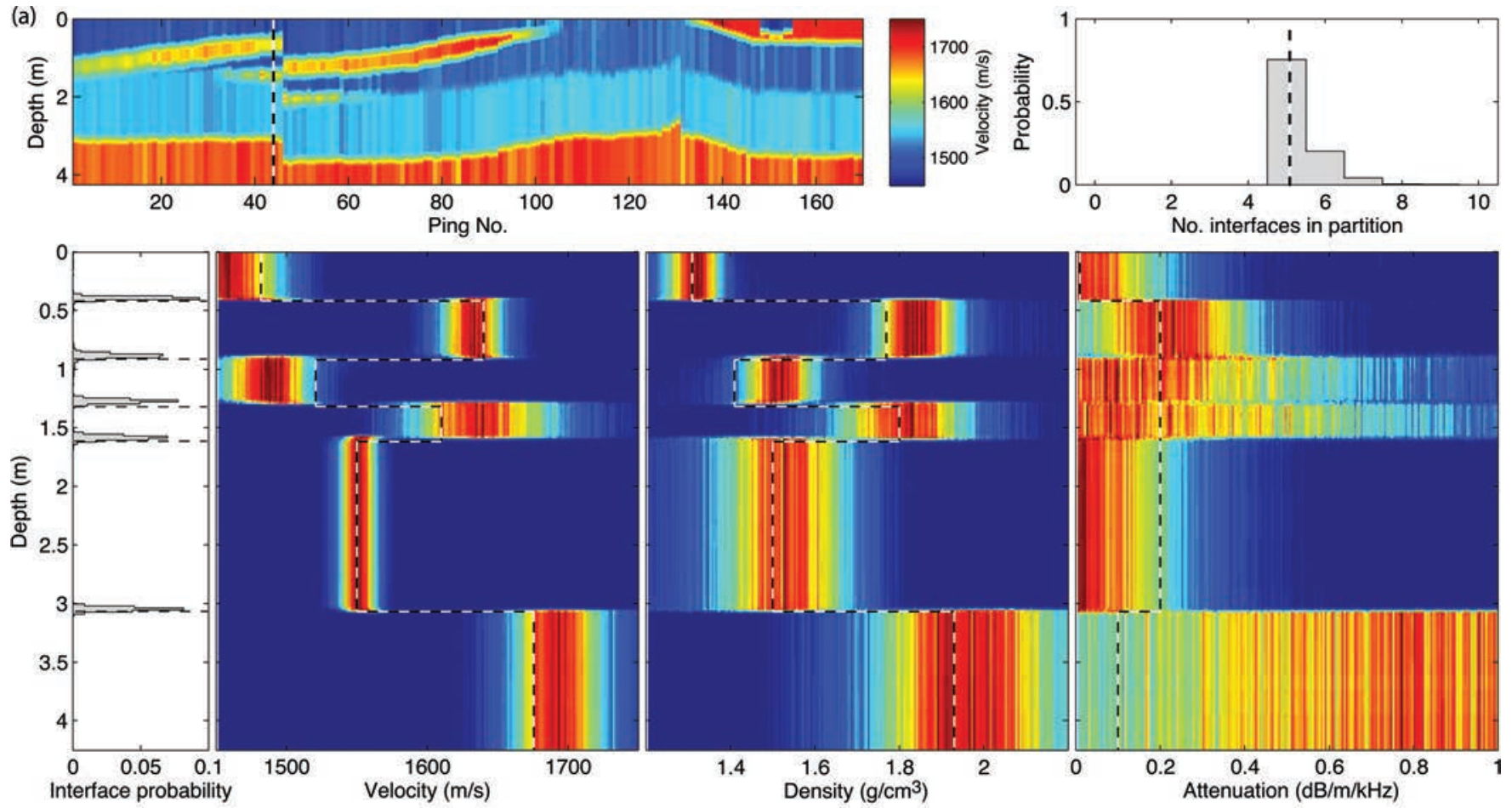

(b)
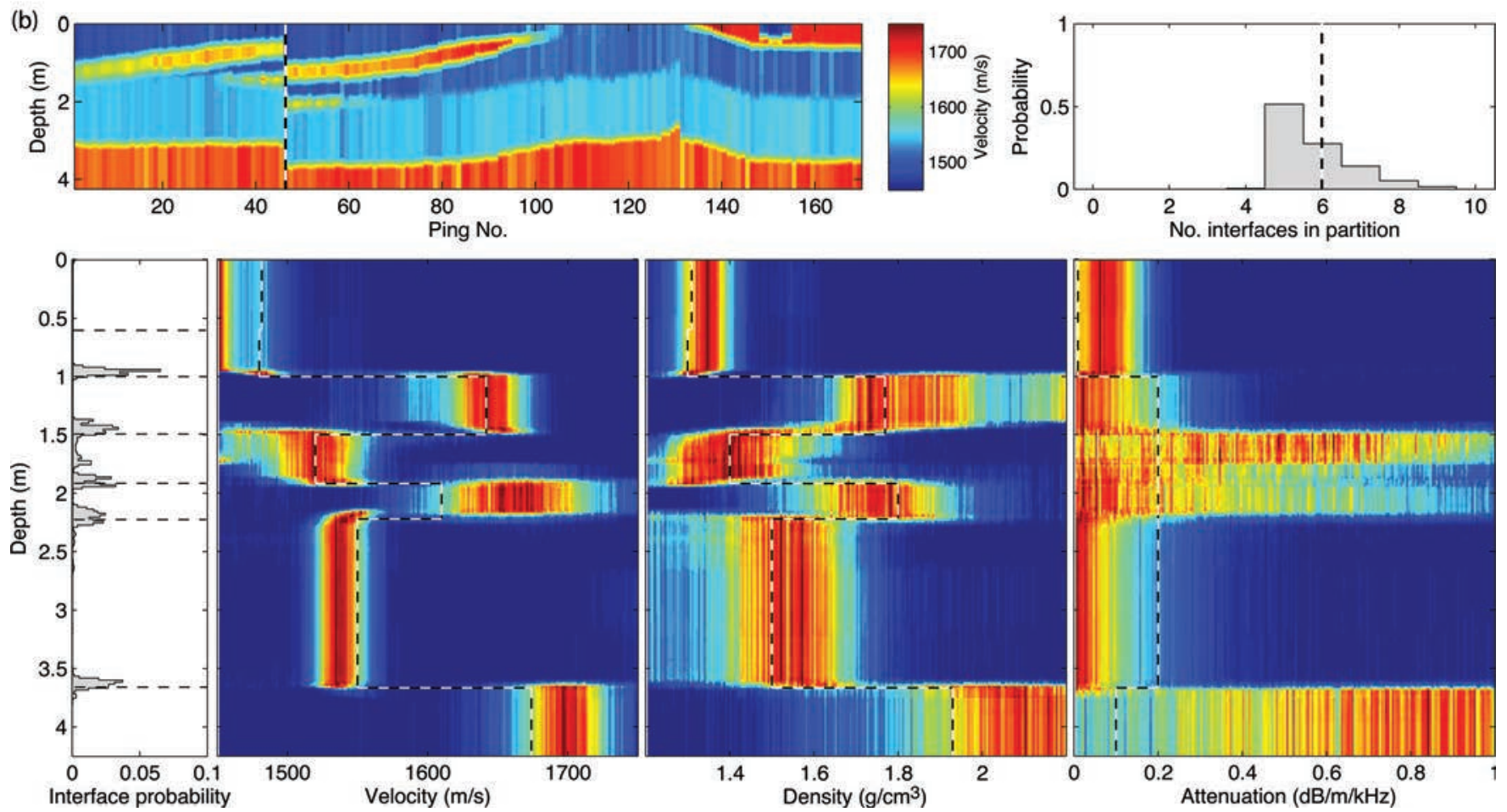

FIG. 8. (Color online) Profile marginal distributions for (a) ping 44 before the fault and (b) ping 46 immediately after the fault. True parameter values are given as dashed lines and the location of the pings is indicated as a dashed line along the track. 
uncertainties and inter-relationships. Figure 8 shows a detailed analysis of the parameter uncertainty (a) just before and (b) just after crossing the geologic fault. Geoacoustic parameter uncertainties are shown in terms of marginal profile distributions that illustrate the uncertainty of geoacoustic parameters as a function of depth. Profile marginals are considered for velocity, density, and attenuation. The true environment is given as a dashed line in the profile marginals. Further, the location of the results for the pings is marked as a dashed line along the track to emphasize that such posterior analysis can be carried out for any ping of interest. In addition, interface probability marginals are plotted as a function of depth, quantifying the probability of interface locations. Interface probability marginals such as these are the basis for interface probability maps (Fig. 6). Marginal distributions of the number of interfaces in the partition show the support of the data for the different model parametrizations. The number of interfaces detected by the trans-dimensional SMC algorithm does not change when the fault is crossed since the velocity and density contrasts are very small between the topmost sediment before the fault and the topmost sediment after the fault. However, the interface probability in Fig. 8(b) shows a wider spread and the profile marginals show some complicated (multi-modal) structure that is likely due to the difficulty the fault poses for algorithm convergence. However, the results quickly stabilize over the next few pings following the fault.

The overall uncertainty of the inversion as a function of depth and ping number is represented in Fig. 9 as a map of the width of the 95\% HPD credibility intervals for (a) velocity, (b) density, and (c) attenuation. It should be noted that the high uncertainties along layer interfaces in Fig. 9 are artifacts due to the computation of the HPD credibility intervals. The width of the HPD is large when a layer interface with a sharp contrast is encountered. Several observations typical for reflection-coefficient inversion can be made. Parameter uncertainty is generally higher in thin (relative to wavelength) layers, which is particularly prominent for the velocity structure but can also be seen clearly for attenuation uncertainty. The uncertainty for all parameters in the underlying half-space is high when compared to layers that are bound by two reflecting interfaces. The uncertainty in density parameters generally increases with depth and is large for layers underlying a layer with high impedance. The opposite effect of low impedance sediments provides a "window" into the sediment that is clearly illustrated by low uncertainties in the neighborhood of ping 120 [particularly Fig. 9(b)]. In addition, parameter uncertainty is smaller when the amount of structure in the true environment is small (few layers), which is also clearly visible around ping 120 , where $k=2$ and the sediment has relatively low impedance until the half-space is encountered at $\sim 3-\mathrm{m}$ depth, resulting in low uncertainty.

Finally, to study the effect of data quality along the track, the simulation and inversion were repeated for the same environment (Fig. 3), but a different data-error standard deviation was applied to the data at each ping. The standard deviation generally varied smoothly along the track [Fig. 10(b)] but increased abruptly several times to simulate poor data quality for some pings. The range of standard deviation used to simulate the data was between 0.025 and 0.046. In this case, the application of Eq. (21) is particularly important for the efficiency of the algorithm and the quality of the results since likelihood values of the inversions can change significantly between pings [Fig. $10(c)$. Inversion of the whole track was almost a factor of 2 slower in this case since converging to a new target can be challenging due to changes in data quality even when

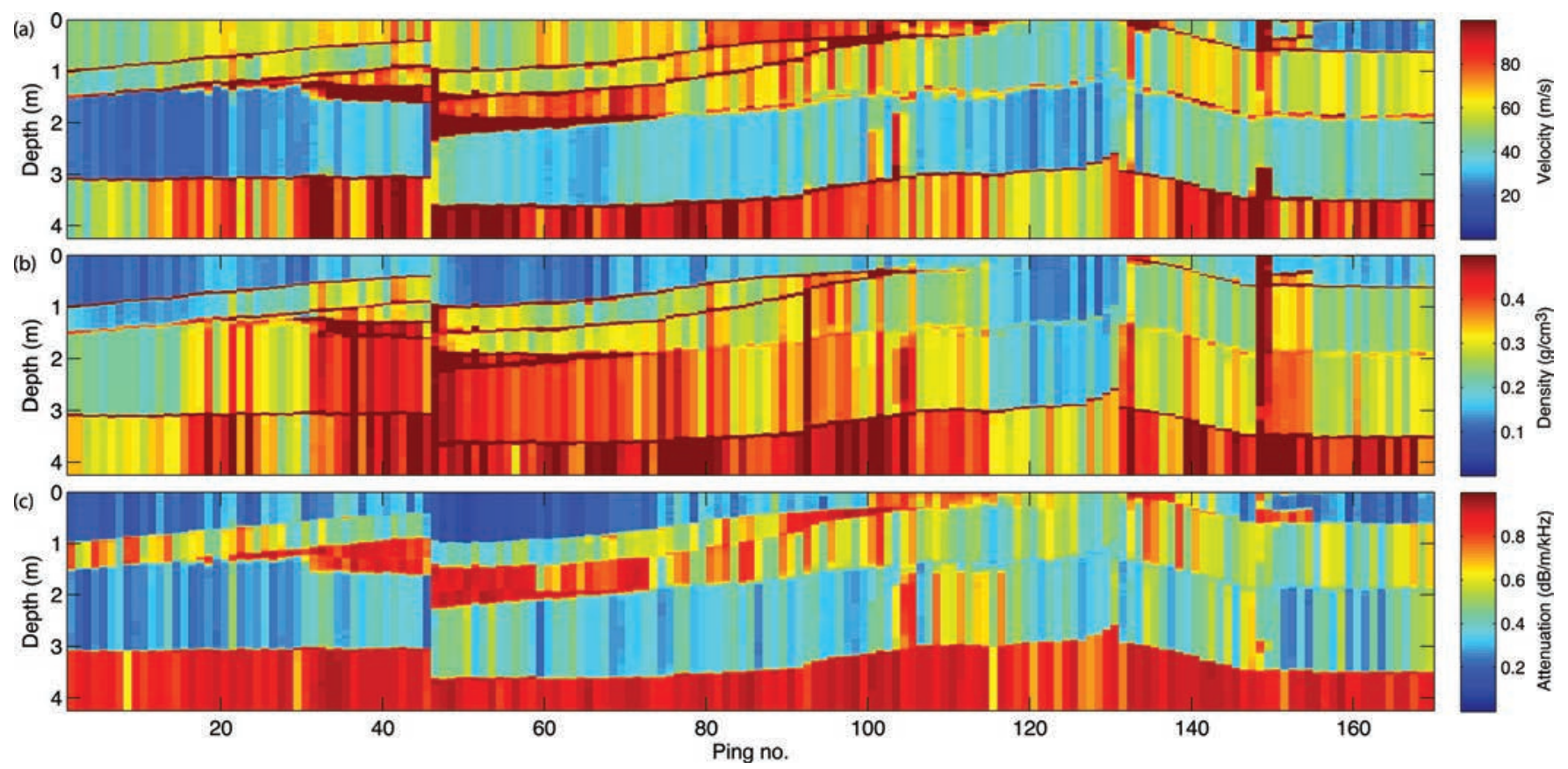

FIG. 9. (Color online) Map width of 95\% HPD credibility interval widths as a function of depth and ping number for velocity, density, and attenuation in (a)-(c), respectively. 

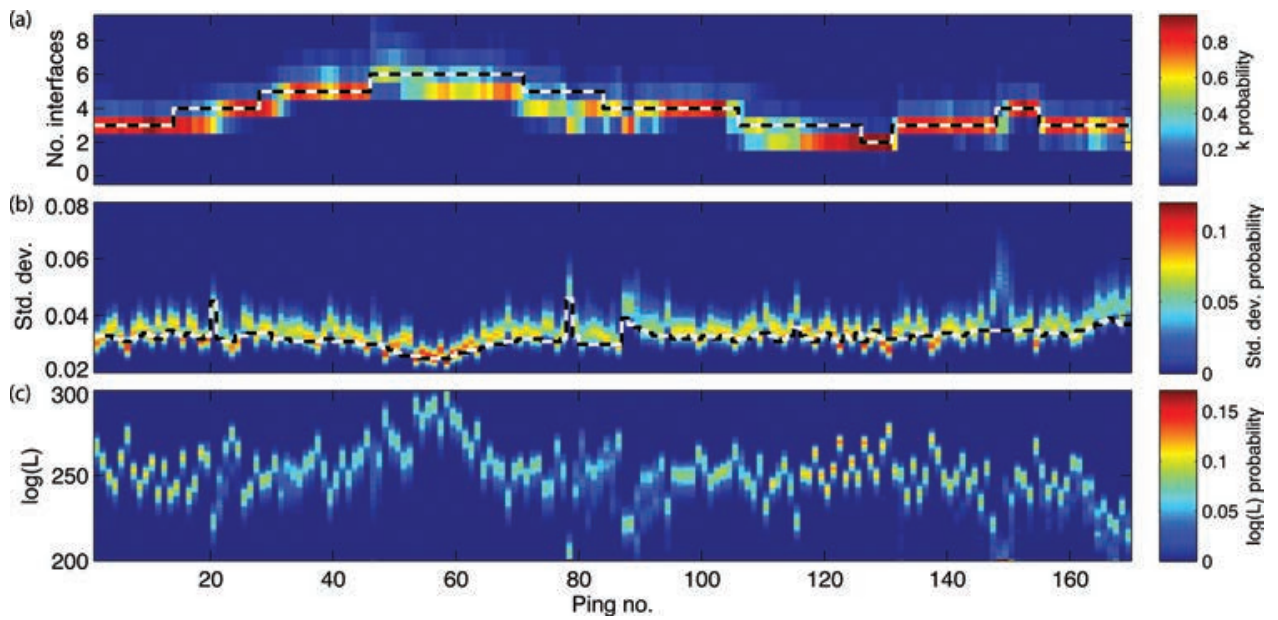

FIG. 10. (Color online) Marginal probability distributions along the track for (a) number of interfaces and (b) data standard deviation (true number of layers and standard deviations are given as dashed lines). (c) The log-likelihood along the track for the case where data errors change from ping to ping.

the environment does not significantly change. However, the use of bridging distributions resulted in comparable quality of inversion results without any other changes to the algorithm.

\section{SUMMARY AND DISCUSSION}

This paper developed a sequential Monte Carlo algorithm for trans-dimensional dynamic Bayesian systems. Such systems are commonly encountered in geoacoustic inversion applications where the unknown environmental structure is more complex than the data information content can support. In such situations, it is advantageous to use the natural parsimony intrinsic to Bayes' theorem and let the data determine the amount of seabed structure that is supported. This issue is particularly important when the lateral spatial variability of sediments is of interest. The problem is addressed here by considering the inverse problem as sequential $^{18}$ and trans-dimensional. ${ }^{14}$ The sequential Monte Carlo algorithm applies elements of importance sampling, simulated annealing, bridge sampling, and reversible jump Markov chains ${ }^{15}$ to track a complicated simulated sediment environment that includes gradual changes as well as large abrupt changes such as geological faults and erosional channels. For efficient inversion of the 170 data sets along the track considered here, the basic bootstrap particle filter ${ }^{17}$ is augmented by bridging distributions ${ }^{13,26}$ before the resampling step to allow the SMC algorithm to quickly find highlikelihood regions when new data are introduced to the algorithm. The bridging distributions are implemented using AIS (Refs. 12 and 13) which provides an efficient approach to obtain a weighted sample of the filter distribution for difficult problems. Difficult problems are encountered when the data information content is high (peaked likelihoods) or when the information content changes rapidly between pings. In such cases, the high posterior probability region of interest at the current step can be far from the corresponding region at previous steps and these regions can be separated by extensive low likelihoods. Particle filters that do not apply MCMC steps may fail in such cases, and particle filters that only include simple MCMC steps ${ }^{20}$ may require very long Markov chains to find the new high-likelihood region, causing prohibitively high computational demands.
After resampling, a trans-dimensional Markov chain is used to further balance the particle cloud to the target distribution. The trans-dimensional chain is implemented using a birth-death scheme with a reversible jump $\mathrm{MH}$ acceptance and a partition model which allows the number of sediment layers to change along the track as determined by the data. In this approach, only the range of the expected number of sediment layers needs to be specified (e.g., $k \in[0,20])$; hence, the required prior information is small and applying the algorithm to complex data sets is straightforward.

The trans-dimensional particle filter inversion algorithm was applied to simulated acoustic data representing reflection coefficients as a function of angle and frequency as would be measured with, e.g., towed HLAs. However, the algorithm is general and can be applied to many different types of data including experimental configurations typically used for matched-field inversion experiments. In the present application, the simulated seabed environment consisted of a layered model of sound velocity, density, and attenuation with lateral variations in geoacoustic parameters within layers and changes in the number of layers. The sequential algorithm successfully recovered the geoacoustic parameter estimates and probability distributions along the track using 10000 particles. The relatively large number of particles was chosen to track of the complicated, multi-modal, trans-dimensional distributions of high dimension, but could be chosen much lower when the primary interest is not in the filtering distributions but rather some of its moments [e.g., parameter MAP or (ensemble) posterior mean estimates and standard deviations]. Computational cost scales approximately linearly with the number of particles. The algorithm also inverts for the data-error standard deviation along the track and hence can handle tracks where data quality changes significantly. The computational cost increases due to the added complexity of such inversions but can handle the problem without any other changes to the algorithm and without the need to introduce other prior knowledge.

Although detailed benchmarking was not carried out, the computational time required for geoacoustic inversion along the whole track was a small fraction of what would 
have been needed to analyze each data set individually (estimated to be at least 2 orders of magnitude longer). A simultaneous inversion of all data for all parameters would not have been possible with the computational facilities available to the authors.

\section{ACKNOWLEDGMENTS}

The authors gratefully acknowledge the support of an Office of Naval Research (N00014-09-1-0394) grant. The computational work was carried out on a parallel high-performance computing cluster operated by the authors at the University of Victoria funded by the Natural Sciences and Engineering Research Council of Canada and the Office of Naval Research. The authors thank Michael J. Wilmut and two anonymous reviewers for helpful comments and improving the quality of the manuscript.

${ }^{1}$ J. Dettmer, S. E. Dosso, and C. W. Holland, "Joint time/frequencydomain inversion of reflection data for seabed geoacoustic profiles," J. Acoust. Soc. Am. 123, 1306-1317 (2008)

${ }^{2}$ C. W. Holland, J. Dettmer, and S. E. Dosso, "Remote sensing of sediment density and velocity gradients in the transition layer," J. Acoust. Soc. Am. 118, 163-177 (2005).

${ }^{3}$ S. E. Dosso, "Quantifying uncertainty in geoacoustic inversion. I. A fast Gibbs sampler approach,” J. Acoust. Soc. Am. 111, 129-142 (2002).

${ }^{4}$ D. J. Battle, P. Gerstoft, W. S. Hodgkiss, W. A. Kuperman, and P. L. Nielsen, "Bayesian model selection applied to self-noise geoacoustic inversion," J. Acoust. Soc. Am. 116, 2043-2056 (2004).

${ }^{5}$ Y. Jiang, N. R. Chapman, and H. A. DeFerrari, "Geoacoustic inversion of broadband data by matched beam processing," J. Acoust. Soc. Am. 119, 3707-3716 (2006).

${ }^{6}$ C.-F. Huang, P. Gerstoft, and W. S. Hodgkiss, "Uncertainty analysis in matched-field geoacoustic inversions," J. Acoust. Soc. Am. 119, 197-207 (2006).

${ }^{7}$ M. Ballard, K. Becker, and J. Goff, "Geoacoustic inversion for the New Jersey shelf: 3-D sediment model,” IEEE J. Ocean. Eng. 35, 28-42 (2010). ${ }^{8}$ M. Fallat, "Geoacoustic characterization of a range-dependent environment," Ph.D. thesis, School of Earth and Ocean Sciences, University of Victoria, Victoria, 2004, pp. 1-123.

${ }^{9}$ M. Siderius, P. L. Nielsen, and P. Gerstoft, "Range-dependent seabed characterization by inversion of acoustic data from a towed receiver array," J. Acoust. Soc. Am. 112, 1523-1535 (2002).

${ }^{10}$ J. Dettmer, C. W. Holland, and S. E. Dosso, "Analyzing lateral seabed variability with Bayesian inference of seabed reflection inversions," J. Acoust. Soc. Am. 126, 56-69 (2009).

${ }^{11}$ J. Dettmer, S. E. Dosso, and C. W. Holland, "Model selection and Bayesian inference for high resolution seabed reflection inversion," J. Acoust. Soc. Am. 125, 706-716 (2009).

${ }^{12}$ J. Dettmer, S. E. Dosso, and J. C. Osler, "Bayesian evidence computation for model selection in geoacoustic inversion," J. Acoust. Soc. Am. 128, 3406-3415 (2010).

${ }^{13}$ R. M. Neal, “Annealed importance sampling,” Stat. Comput. 11, 125-139 (2001).

${ }^{14}$ J. Dettmer, S. E. Dosso, and C. W. Holland, "Trans-dimensional geoacoustic inversion," J. Acoust. Soc. Am. 128, 3393-3405 (2010).

${ }^{15} \mathrm{P}$. J. Green, "Reversible jump Markov chain Monte Carlo computation and Bayesian model determination," Biometrika 82, 711-732 (1995).

${ }^{16} \mathrm{~T}$. Bodin and M. Sambridge, "Seismic tomography with the reversible jump algorithm,” Geophys. J. Int. 178, 1411-1436 (2009).
${ }^{17}$ N. Gordon, D. Salmond, and A. Smith, "Novel approach to nonlinear/non-Gaussian Bayesian state estimation,” IEEE Proc. Radar Signal Process. 140, 107-113 (1993)

${ }^{18}$ A. Doucet, N. de Freitas, and N. Gordon, editors, Sequential Monte Carlo in Practice (Springer, New York, 2001), pp. 1-581.

${ }^{19} \mathrm{C}$. Berzuini and W. Gilks, "Resample-move filtering with cross-model jumps," in Sequential Monte Carlo in Practice, edited by A. Doucet, N. de Freitas, and N. Gordon (Springer, New York, 2001), pp. 117138.

${ }^{20}$ W. R. Gilks and C. Berzuini, "Following a moving target-Monte Carlo inference for dynamic Bayesian models," J. R. Stat. Soc. 63, 127-146 (2001).

${ }^{21}$ C. Yardim, P. Gerstoft, and W. S. Hodgkiss, "Geoacoustic and source tracking using particle filtering: Experimental results," J. Acoust. Soc. Am. 128, 75-87 (2010).

${ }^{22}$ A. Gelfand and A. Smith, "Sampling-based approaches to calculating marginal densities," J. Am. Stat. Assoc. 85, 398-409 (1990).

${ }^{23}$ A. F. M. Smith, "Bayesian computational methods," Philos. Trans. R. Soc. London 337, 369-386 (1991).

${ }^{24}$ W. R. Gilks, S. Richardson, and D. J. Spiegelhalter, editors, Markov Chain Monte Carlo in Practice: Interdisciplinary Statistics (Chapman \& Hall/CRC, Boca Raton, FL, 1996), pp. 1-486.

${ }^{25}$ C. Yardim, P. Gerstoft, and W. S. Hodgkiss, "Tracking of geoacoustic parameters using Kalman and particle filters," J. Acoust. Soc. Am. 125, 746-760 (2009).

${ }^{26} \mathrm{~S}$. Godsill and T. Clapp, "Improvement strategies for Monte Carlo particle filters," in Sequential Monte Carlo in Practice, edited by A. Doucet, N. de Freitas, and N. Gordon (Springer, New York, 2001), pp. 139-158.

${ }^{27}$ D. B. Rubin, "Using the SIR algorithm to simulate posterior distributions," in Bayesian Statistics 3, edited by J. M. Bernardo, M. H. DeGroot, D. V. Lindley, and A. F. M. Smith (Oxford University Press, Oxford, 1988), pp. 99-171.

${ }^{28}$ A. Smith and A. Gelfand, "Bayesian statistics without tears: A samplingresampling perspective," Am. Stat. 46, 84-88 (1992).

${ }^{29}$ M. K. Pitt and N. Shephard, "Filtering via simulation: Auxiliary particle filters," J. Am. Stat. Assoc. 94, 590-599 (1999).

${ }^{30}$ S. Kirkpatrick, C. D. Gelatt, and M. P. Vecchi, "Optimization by simulated annealing," Science 220, 671-679 (1983).

${ }^{31}$ P. J. Green, "Trans-dimensional Markov chain Monte Carlo," in Highly Structured Stochastic Systems, Oxford Statistical Science Series, edited by P. J. Green, N. L. Hjort, and S. Richardson (Oxford University Press, Oxford, 2003), pp. 179-198.

${ }^{32}$ R. C. Aster, B. Borchers, and C. H. Thurber, Parameter Estimation and Inverse Problems, International Geophysics (Elsevier, Amsterdam, the Netherlands, 2005), pp. 395-402.

${ }^{33}$ T. Bodin, M. Sambridge, and K. Gallagher, "A self-parametrizing partition model approach to tomographic inverse problems," Inverse Probl. 25, 055009 (2009).

${ }^{34}$ D. G. T. Denison, C. C. Holmes, B. K. Mallick, and A. F. M. Smith, Bayesian Methods for Nonlinear Classification and Regression (Wiley, New York, 2002), pp. 1-277.

${ }^{35}$ W. Press, S. Teukolsky, W. Vetterling, and B. Flannery, Numerical Recipes in Fortran 77, 2nd ed. (Cambridge University Press, Cambridge, 1997), pp. 1-933.

${ }^{36} \mathrm{C}$. W. Holland and J. Osler, "High-resolution geoacoustic inversion in shallow water: A joint time- and frequency-domain technique," J. Acoust. Soc. Am. 107, 1263-1279 (2000).

${ }^{37}$ E. L. Hamilton, "Variations of density and porosity with depth in deep-sea sediments," J. Sediment. Petrol. 46, 280-300 (1976).

${ }^{38}$ L. M. Brekhovskikh and O. A. Godin, Acoustics of Layered Media I: Plane and Quasi-Plane Waves, 1st ed., Wave Phenomena (Springer, New York, 1990), pp. 203-238.

${ }^{39}$ A. Papoulis and S. U. Pillai, Probability, Random Variables, and Stochastic Processes (McGraw-Hill, New York, 2002), pp. 1-852. 